\title{
VARIABLES IMPACTING GFR ESTIMATION METHOD FOR DRUG DOSING IN CKD: ARTIFICIAL NEURAL NETWORK PREDICTION MODEL
}

\author{
SABA M. AIJASMI ${ }^{12}{ }^{*}$, AMER H. KHAN ${ }^{*}$, SYED AZHAR SYED SULAIMAN ${ }^{1}$, MIRZA R. BAIG ${ }^{3}$, MALIK OBAIDULLAH ${ }^{4}$, \\ EMAD A. MAKRAMALLA ${ }^{2}$
}

${ }^{1}$ Discipline of Clinical Pharmacy, School of Pharmaceutical Sciences, Universiti Sains Malaysia, 11800 Penang, Malaysia, ${ }^{2}$ Department of

Pharmacy, Zayed Military Hospital, Abu Dhabi, United Arab Emirates, ${ }^{3}$ Clinical Pharmacy and Pharmacy Practice Department, Dubai Pharmacy College, Dubai, United Arab Emirates, ${ }^{4}$ Drug Regulatory Authority, Ministry of Health Pakistan, Islamabad, Pakistan

Email: saba.aljasmi@msc.mil.ae

Received: 15 Sep 2019, Revised and Accepted: 07 Nov 2019

\section{ABSTRACT}

Objective: This study aimed to measure concordance between different renal function estimates in terms of drug doses and determine the potential significant clinical differences.

Methods: Around one hundred and eighty patients $\geq 18$ y) with chronic kidney disease (CKD) were eligible for inclusion in this study. A pairedproportion cohort design was utilized using an artificial intelligence model. CKD patients refined into those who have drugs adjusted for renal function. For superiority of Cockcroft-Gault (CG) vs. modified diet in renal disease (MDRD) guided with references for concordance or discordance of the two equations and determined the dosing tiers of each drug. Validated artificial neural networks (ANN) was one outcome of interest. Variable impacts and performed reassignments were compared to evaluate the factors that affect the accuracy in estimating the kidney function for a better drug dosing.

Results: The best ANN model classified most cases to CG as the best dosing method (79 vs. 72). The probability was $85 \%$ and the top performance was slightly above $93 \%$. Creatinine levels and CKD staging were the most important factors in determining the best dosing method of CG versus MDRD. Ideal and actual body weights were second (24\%). Whereas drug class or the specific drug was an important third factor (14\%).

Conclusion: Among many variables that affect the optimal dosing method, the top three are probably CKD staging, weight, and the drug. The contrasting CKD stages from the different methods can be used to recognize patterns, identify and predict the best dosing tactics in CKD patients.

Keywords: Artificial, Neural networks, Cockcroft-gault, Modification of diet in renal disease, Chronic kidney disease, Clinical response

(C) 2019 The Authors. Published by Innovare Academic Sciences Pvt Ltd. This is an open-access article under the CC BY license (http://creativecommons.org/licenses/by/4.0/) DOI: http://dx.doi.org/10.22159/ijpps.2019v11i12.35688

\section{INTRODUCTION}

Starting in 1998, the United States FDA made a requirement that new medication applications include renal dosing [1]. In 2004, the European Medicines Agency (EMEA) brought forth a similar statement in acute kidney injury patients [2]. Many of the old medications still have dosing algorithms largely based on postmarketing data [3]. Patients with CKD have several challenges that may alter the response to medications, and hence, may affect the optimal dose of medications.

Dosing of medications in CKD patients is further complicated by the very nature of the dose-response interaction for the various medications. Some medications need high peak levels, whereas others require less fluctuation of the levels over the dosing intervals [4-18]. For example, aminoglycoside antibiotics are an example of the former, whereas $\beta$-lactams are a representative of the latter [19-22].

Artificial neural networks (ANN) employed to predict if choosing the optimal renal function estimate to dose drugs in CKD patients is heuristic rather than an exact problem. Therefore, we are certain that a good ANN model will very likely give better predictive ability than simple statistical pattern recognition. The ANN model incorporated one outcome; namely, superior renal function estimate (CG or MDRD). Therefore, our actual ANN structure would look like the one shown in fig. 1 . This is a probabilistic neural network with 24-input layer, two hidden layers; one for the study cases, and the second for two categories of the outcome and a final output layer. Generalized regression networks (GRN) used for continuous outcomes and make predictions that can be drawn against actual data (fig. 2). The first hidden layer in both designs ensures accuracy. The second hidden layer reduces dimensionality to drive ANN toward fast convergence $[23,24]$. Using ANN to try to maximize the accuracy, by which we can dose drugs with either CG or MDRD right from the beginning, will give us an idea about some of the factors that may be used as input variables in an ANN model. ANN model will then determine which variables are of the greatest importance for the prediction of the best renal function estimates.

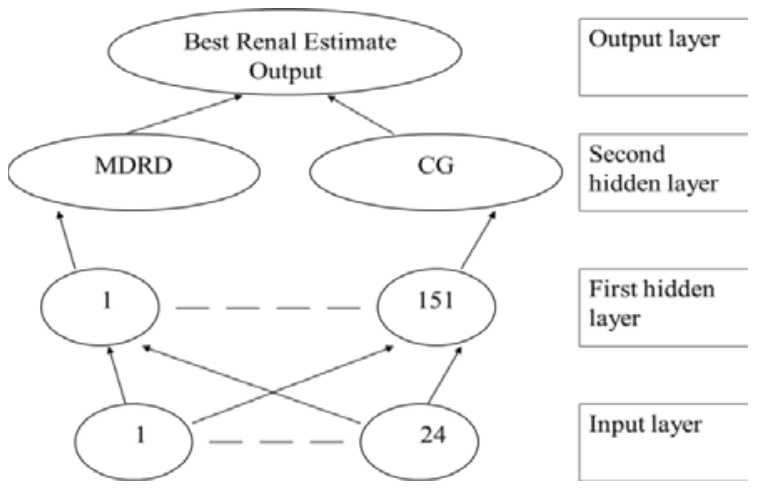

Fig. 1: Our ANN model structure

\section{MATERIALS AND METHODS}

Study design and setting

A paired-proportion cohort design was utilized using an artificial intelligence model. The study was conducted at a national military hospital in the United Arab Emirates (UAE) with a 300-bed tertiary care center serving around 300,000 patients. The prevalence of CKD in UAE population is estimated at about $10 \%$. For the purposes of this 
study, we will use the term "pattern" to mean an individual patient case with its distinct clinical input variables. Diseases as input variables were based on actual diagnoses documented in the patient files or the standardized study data collection sheets. Almost all numerical values were real values for the patients with missing values comprising a very minute less than $1 \%$ replaced with median of the variable.

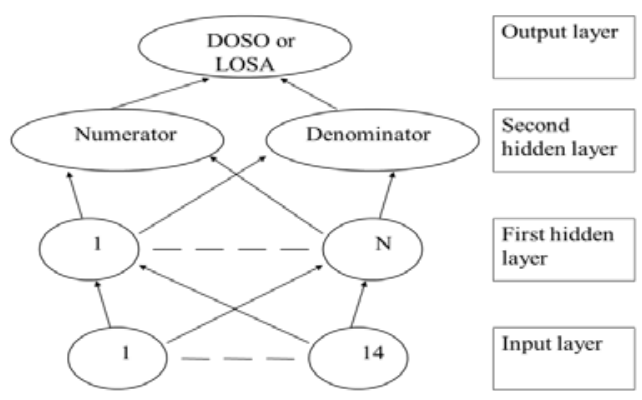

Fig. 2: ANN-GRN example
This study was undertaken in full accordance with the declaration of Helsinki 1964 as all its amendments. An informed consent was obtained from every patient before their enrollment in the study. Moreover, the ethical study approvals were secured from relevant institutions.

\section{Study subjects}

All adult, patient's $\geq 18 \mathrm{y}, \mathrm{CKD}$ (based on physician/nephrologistsassigned diagnosis) were eligible for inclusion in the current study. Patients who were pregnant had cancer, human immunodeficiency virus (HIV), acute kidney failure, dialysis, and those on medications interfering with serum creatinine (SCr) were excluded. As shown in table 1 , a cohort of 180 consecutive cases was included in the study to provide the desirable clinical response in each case, variables included in the ANN model. Recruited CKD patients would be refined into those who have drugs adjusted for renal function and were added as an input variable (table 2). For superiority of CG vs. MDRD and guided with references such as Lexi-comp®, adult drug bookkidney disease program, package insert or European Medicines Agency (EMC) monographs $[25,26]$. Our research determined the dosing tiers of each drug. Consequently, our group determined the presence of concordance or discordance of the two equations.

Ethical considerations

Table 1: Variables included in the ANN model and their summary data*

\begin{tabular}{|c|c|}
\hline Variable & Description \\
\hline \multicolumn{2}{|l|}{ Input } \\
\hline Age & 67 (23-99)DrugsBisoprolol (22) \\
\hline ABW & 76 (40.5-115) Trimetazidine (11) \\
\hline Gender & M: (107), F: (44) Atenolol (9) \\
\hline IBW & 59 (45-84) Sitagliptin/Metformin(8) \\
\hline ABW to IBW & $1.32(0.5-2.18)$ Sitagliptin (5) \\
\hline Height & 164 (75-184) Metformin (4) \\
\hline $\mathrm{SCr}$ & $1.69(0.97-6.57)$ Others $(8)$ \\
\hline $\mathrm{CG} \mathrm{eCrCl}$ & 31.0 (10.4-59.7)CG Stage3A (35), 3B (42), 4 (65), 5 (9) \\
\hline MDRD eGFR & $\begin{array}{l}42.7(2.13-75.0) \text { MDRD Stage } 3 A(42), 3 B(67), 4(18), 5(4) \\
\text { mGFR Stage 3A (18), 3B (60), } 4(21), 5(5)\end{array}$ \\
\hline No of Drugs & 8 (3-26)Doses Adjusted Yes (42), No (109) \\
\hline DM & Yes (90), No (61) Electrolyte Disturb. Yes (39), No (112) \\
\hline HTN & Yes (137), No (14)CHD Yes (45), No (106) \\
\hline DLP & Yes (112), No (39)Polypharmacy Yes (135), No (16) \\
\hline Smoker & Yes (3), No (148)Matching Mgfr Yes (45), No (85) \\
\hline Anemia & Yes (48), No (103) \\
\hline \multicolumn{2}{|l|}{ Output } \\
\hline Estimate Dosing Response & CG (85), MDRD (66) \\
\hline
\end{tabular}

ABW; actual body weight, IBW; ideal body weight, SCr; serum creatinine, DM; diabetes mellitus, HTN, hypertension, DLP; dyslipidemia, CHD; congenital heart disease *Continuous variables as Median (Range), Categoric variables as group (count)

Table 2: Most common drugs as an input variable

\begin{tabular}{ll}
\hline Drug & Count \\
\hline Bisoprolol & 22 \\
Trimetazidine & 11 \\
Atenolol & 9 \\
Sitagliptin/Metformin & \\
Sitagliptin & \\
Metformin & 8 \\
Others & 5 \\
\end{tabular}

\section{Sample size}

For the primary parameter of interest which was matched the proportion of the superiority of CG vs. MDRD, a free online tool was used, namely, (Satulatorbeta), which was accessed on January 11th, 2019 (URL: http://statulator.com/SampleSize/ss2PP.html) to calculate the required sample size. Assuming that $45 \%$ and $55 \%$ of the pairs are superior for either CG or MDRD, respectively, the correlation between paired observation was 99\% (almost 100\%) and after applying continuity correction, the study would require a sample size of 19 pairs to achieve a power of $80 \%$ and a two-sided significance of $5 \%$ for detecting a difference of 0.10 between marginal proportions. Predicting a dropout rate of about 20\%, 24 such pairs were required to detect the $10 \%$ difference.

\section{Artificial neural network model}

We constructed our ANN model by using Microsoft Excel add-in (Microsoft Corp., Redmond, WA), Neural Tools, version 7.6.0 
(Palisade Corp., Ithaca, NY). Input nodes consisted of 15 categorical and 9 continuous variables. We replaced missing input values $(<1 \%)$ with medians and the most common classes for continuous and categorical variables, respectively. The ANN model incorporated one outcome; namely, superior renal function estimate (CG or MDRD). Therefore, our actual ANN structure would look something like that shown in fig. 1 . This is a probabilistic neural network with 24-input layer, two hidden layers; one for the 151 cases, and the second for two categories of the outcome and a final output layer. Our combined dataset of 151 cases was divided as follows: 8 cases for validation or live prediction, and the remaining cases randomly assigned to training (80\%) or internal validation or testing $(20 \%)$. Live prediction or validation cases are cases that our ANN did not encounter before, i.e. the outputs in these cases are not known to the ANN. Training data are known and used in the training and fitting of the ANN. Whereas the testing or internal validation dataset is data known to the ANN which the ANN does not use in the training but rather it uses it in optimizing the model to new data with known output. The $80 / 20$ division of the data training/testing gave the best ANN results. The $20 \%$ testing set prevents a problem known as overtraining or over-fitting, which is simply an ANN that is just trained to classify known data but not new one. We enabled all possible stop conditions: $2 \mathrm{~h}$ of training, 1,000,000 trials, and error change of less than $1 \%$ within 60 min. Neural Tools version 7.6.0 generated variable impact (VI) for each input used in the training and validation of a given ANN. VI is a percentage that represents overall contribution of a given variable to the predicted outcome in the model. Furthermore, we reassigned inputs to study the effect of these modified values on outcomes by live predictions. Therefore, we were able to completely describe the ANN and role of individual variables including the drug in our model.

\section{Statistical analyses}

We studied multivariate for all outcome in one of two ways: reassignment of inputs and subgroup analyses. We applied all univariate statistical tests. We applied v2 or Fisher exact tests, as indicated, to evaluate statistical significance in case of unpaired categoric data. McNamara's test used for paired categoric data. Unpaired and paired, 2-tailed, Student t-tests used as indicated to evaluate significance for continuous variables. Nonparametric tests were implemented when normality assumptions failed. A p-value of less than 0.05 was considered statistically significant. All statistical tests were performed by using SPSS, version 15.0.1, for Windows (IBM, Somers, NY). Variables included in the study were identified, measured and evaluated only from the sample population and did not include all possible variables that may affect the optimal dosing method.

\section{RESULTS}

\section{Artificial neural network model pearls}

\section{ANN model convergence}

Here Our ANN training converged to the best model after 261 trials (epochs) and in less than 1 minute. Actual numbers of cases used in training, testing and validation were 114,29 , and 8, respectively. Error reduction beyond $1 \%$ ceased and resulted in the auto-stop of the training process.

\section{Best ANN performance}

Our best ANN model performed to a quite acceptable level of accuracy. First for training data, the ANN model performed completely accurate with less zero cases with the probability of more than $80 \%$ of incorrect predictions. As far as testing and prediction data, the model performed quite favorably to no model with only $22 \%$ of bad predictions at a probability of more than $80 \%$. Overall performance for the entire dataset is accurate to $91 \%$ of the cases. So the best ANN model can correctly classify the case into the best renal function estimate in $91 \%$ of the scenarios. The net would still classify most cases to CG as the best dosing method (79 vs. 72).

\section{Effect of varying the probability of prediction on the accuracy of the prediction}

Varying the probability of prediction, we were able to identify the cutoff probability at which the ANN performance is maximized. This probability was $85 \%$ and the top performance was slightly above $93 \%$.

\section{Variable impacts}

Creatinine levels and CKD staging were the most important factors in determining the best dosing method CG versus MDRD (combined VI of above 51\%). Ideal and actual body weights were second (combined VI of 24\%). Whereas drug class or the specific drug was a third important factor (14\%). Comorbidities had an insignificant contribution to the prediction of the best renal estimate (all VI less than 1\%). These findings determining which renal function estimate is linked to the optimal drug dosing in our patient population (fig. 3).

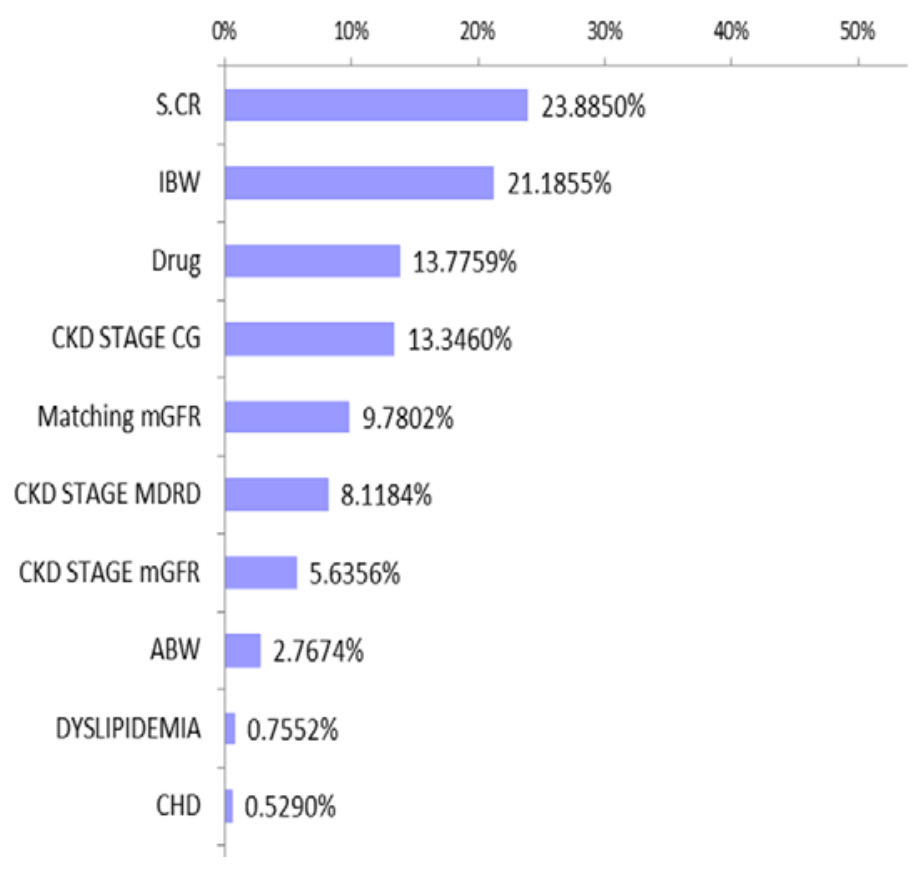

Fig. 3: Variable impacts for the best ANN model 


\section{Reassignment analyses}

We have attempted multiple reassignment analyses to the data to study the effect of the various variables and to know whether their effects are stable or fixed. For example, if we keep all the inputs constant and just change all medications to bisoprolol how will that change the predictions the model make? Or if we feed the patterns we had in the previous sections for CG or MDRD superiority, how will that modify the model predictions? Once again the most stable and significant impact was seen for CKD Stage, followed by weight, and thirdly by drugs. Let us take Bisoprolol as an example. If we consider that all the patients have the drug bisoprolol to adjust, there will be 1 more case requiring dosing by CG to optimal clinical response. In detail, three patients will shift optimal dosing from MDRD to CG and 2 from CG to MDRD. If we consider CKD stage, assume we chose that all patients would have the same CKD stages in pattern 1 (table 3). In this case, there will be 10 patients shifting from CG to MDRD and 19 from MDRD to CG. Changing all patients to morbidly obese resulted in 9 shifting to MDRD and to CG. On a matched case-control McNemar test none of these changes mentioned above reach statistical significance. This means that the input variables are almost in a state of equilibrium.

Table 3: Interesting patterns with exclusive superiority of one renal dose estimation method

\begin{tabular}{|c|c|c|c|c|c|}
\hline Pattern Algorithm ${ }^{\Delta}$ & $\mathbf{N}$ & IBW & CG stage & MDRD stage & Superiority \\
\hline \multicolumn{6}{|l|}{ Phase I ₹ } \\
\hline 1 & 14 & $>56$ & $3 \mathrm{~B}$ & 3 & CG \\
\hline 2 & 4 & $<61$ & $3 \mathrm{~A}$ or 5 & 2 or 4 & MDRD \\
\hline 3 & 4 & $<61$ & 5 & 4 & MDRD \\
\hline \multicolumn{6}{|l|}{ Phase II } \\
\hline 1 & 17 & $>56$ & $3 \mathrm{~B}$ & 3 & CG \\
\hline $2^{*}$ & 1 & $<61$ & $3 \mathrm{~A}$ or 5 & 2 or 4 & MDRD \\
\hline $3^{* *}$ & 0 & $<61$ & 5 & 4 & - \\
\hline
\end{tabular}

N; Number of cases with the pattern, IBW; Ideal Body Weight, HTN; Hypertension, CG CKD Stage; Chronic Kidney Diseases stage according to Cockcroft-Gault equation, MDRD CKD Stage; Chronic Kidney Disease stage according to Modification of Diet in Renal Disease Equation. *The one case found in this pattern was a trimetazidine case with both hypertension and polypharmacy. ${ }^{* *}$ No cases with this pattern were seen in the external validation set.

\section{DISCUSSION}

Pharmacotherapy assessment in chronic renal disease (PAIR) criteria is a validated tool to assess medication safety and use issues in patients with CKD. It is noteworthy that inappropriate dosage comprised more than one-third of the drug-related problems (DRPs) in the PAIR criteria [27]. Another study conducted in the residents of aged care facilities; it has shown that DRPs were mostly dose-related [28]. Previous studies have shown that CG and MDRD perform better with varied scenarios [29-32]. However, the study on meropenem found no difference between CG and MDRD [33]

Readers should note our key findings compared with that of Breton et al. [34]. They simply showed that use of CG is associated with more dose adjustments overall in the population of elderly patients with renal impairment. According to the current findings, these risk factors are male sex, 18 to $65 \mathrm{y}$ old, obesity, hypoalbuminemia, and CKD stages III, IV, V, and sometimes II. Some of these sources of variability were well established and studied as summarized by Lascano and Poggio [35]. However, multiple other factors may be involved as discussed by Cockcroft and Gault [36-38]. Dersch and McCormack correctly concluded that dosage selection in renal patients must be a clinical activity that ought to be individualized [39]. Nevertheless, real-life studies can help determine whether using CG or MDRD is superior empirically. Individual patient response and sound clinical judgment would then pave the way for careful escalation or de-escalation of therapy.

We have shown the patterns of exclusive superiority of CG and others for MDRD. Out of total 151 cases we had about 40 cases (26.5\%) that can be accurately dosed to optimal clinical response with CG or MDRD right from the start. Although this percentage is small, it is quite significant compared to completely chaotic dosing. Our ANN improved dramatically on the above fig. With our best ANN, we can dose slightly above $80 \%$ of testing cases and $75 \%$ of totally new cases with the best renal function estimate. We can actually dose slightly above $93 \%$ of the whole set with the right method immediately without the need for trial and error or continuous shifting between CG and MDRD derived doses. These findings are extremely important as we can now accurately choose the optimal dose and not wait until the $4^{\text {th }}$ or the $5^{\text {th }}$ follow up encounter to reach the right dose. This is the first ANN modeling of the drug dosing problem and the optimal renal function estimate method. With our model, we can be extremely confident about the right dose in CKD patients for the vast majority of cases.

\section{LIMITATIONS}

The study took into consideration some of the comorbidities that may affect the function of the kidney but didn't cover all possible chronic illnesses that may affect kidney function.

The study population is mainly collected over a period of $12 \mathrm{mo}$, no randomization could have been done or stratification to even out all other variables and confounders.

In our study, we focused on the available prediction variables in our population to anticipate the best method to estimate kidney function and adjust drug doses. The results needs to be validated in a more controlled study to generalize it.

We have excluded critically ill patients from the study population as it may undermine the assessment of creatinine clearance, moreover fluctuation in the kidney function is affecting the validity of the methods used.

\section{CONCLUSION}

We are sure that among the many variables that affect the optimal dosing method, the top three are probably CKD staging, weight, and the drug in question. A more sophisticated model is required to factor-in all possible variables in order to reach the most appropriate dose for renal patients.

\section{CONFLICT OF INTERESTS}

All authors declared that there was no conflict of interest

\section{AUTHORS CONTRIBUTIONS}

All authors shared equally in this study.

\section{REFERENCES}

1. US Department of Health and Human Services Food and Drug Administration. Guidance for industry: pharmacokinetics in patients with impaired renal function-study design, data analysis, and impact on dosing and labeling; 1998.

2. European Medicines Agency, Committee for Medicinal Products for Human Use. Note for guidance on the evaluation of the 
pharmacokinetics of medicinal products in patients with impaired renal function; 2004.

3. Zuber K, Liles AM, Davis J. Medication dosing in patients with chronic kidney disease. J Am Acad Physic Assist 2013;26:19-25.

4. Cook AM, Hatton Kolpek J. Augmented renal clearance. Pharmacotherapy 2019;39:346-54.

5. Murali R, Sathyanarayana D, Muthusethupathi MA. Assessment of quality of life in chronic kidney disease patients using the kidney disease quality of life-short form questionnaire in indian population: a community-based study. Asian J Pharm Clin Res 2015;8:271-4.

6. Levey AS, Bosch JP, Lewis JB, Greene T, Rogers N, Roth D. A more accurate method to estimate glomerular filtration rate from serum creatinine: a new prediction equation. Modification of Diet in Renal Disease Study Group. Ann Intern Med 1999;130:461-70.

7. National Kidney Disease Education Program. Chronic kidney disease and drug dosing: information for providers; 2010.

8. Hermsen ED, Maiefski M, Florescu MC, Qiu F, Rupp ME. Comparison of the modification of diet in renal disease and cockcroft gault equations for dosing antimicrobials. Pharmacotherapy 2009;29:649-55.

9. Stevens LA, Nolin TD, Richardson MM, Feldman HI, Lewis $\mathrm{JB}$, Rodby $\mathrm{R}$, et al. Comparison of drug dosing recommendations based on measured GFR and kidney function estimating equations. Am J Kidney Dis 2009;54:33-42.

10. Wargo KA, Eiland EH, Hamm W, English TM, Phillippe HM. Comparison of the modification of diet in renal disease and cockcroft-gault equations for antimicrobial dosage adjustments. Ann Pharmacother 2006;40:1248-53.

11. Golik MV, Lawrence KR. Comparison of dosing recommendations for antimicrobial drugs based on two methods for assessing kidney function: cockcroft-gault and modification of diet in renal disease. Pharmacotherapy 2008;28:1125-32.

12. Jennings S, de Lemos ML, Levin A, Murray N. Evaluation of creatinine-based formulas in dosing adjustment of cancer drugs other than carboplatin. J Oncol Pharm Pract 2010;16:113-9.

13. Spruill WJ, Wade WE, Cobb HH. Comparison of estimated glomerular filtration rate with estimated creatinine clearance in the dosing of drugs requiring adjustments in elderly patients with declining renal function. Am J Geriatr Pharmacother 2008;6:15360.

14. Thummel K, Shen D, Isoherranen N. Design and optimization of dosage regimens: pharmacokinetic data. In: Hardman J, Limbird L, Goodman G. (eds). Goodman and Gilman's The Pharmacological Basis of Therapeutics. 11th ed. McGraw-Hill: New York, NY; 2006.

15. Matzke GR, Dowling T. Dosing concepts in renal dysfunction. In: Murphy JE. ed. Clinical Pharmacokinetics Pocket Reference. 5th ed. American Society of Health-System Pharmacists: Bethesda, MD; 2011.

16. Charpentier G, Riveline JP, Varroud Vial M. Management of drugs affecting blood glucose in diabetic patients with renal failure. Diabetes Metab 2000;26:73-85.

17. Snyder RW, Berns JS. Use of insulin and oral hypoglycemic medications in patients with diabetes mellitus and advanced kidney disease. Semin Dial 2004;17:365-70.

18. Rocha A, Almeida M, Santos J, Carvalho A. Metformin in patients with chronic kidney disease: strengths and weaknesses. J Nephrol 2013;26:55-60.

19. Stabler SN, Ensom MH. Extended-interval aminoglycoside therapy for adult patients with febrile neutropenia: a systematic review. Can J Hosp Pharm 2011;64:182-91.

20. Jenh AM, Tamma PD, Milstone AM. Extended-interval aminoglycoside dosing in pediatrics. Pediatr Infect Dis J 2011;30:338-9.
21. Habayeb H, Grundy C, Rangaiah J, Van de Velde S. Continuous beta-lactam intravenous antibiotic infusions for outpatient parenteral antimicrobial therapy. Int J Antimicrob Agents 2018;52:436-7.

22. Falagas ME, Vardakas KZ. Continuous or extended intravenous administration of $\beta$-lactam antibiotics. Am J Respir Crit Care Med 2017;195:1077-8.

23. Saadah LM, Chedid FD, Sohail MR, Nazzal YM, Al Kaabi MR Rahmani AY. Palivizumab prophylaxis during nosocomial outbreaks of the respiratory syncytial virus in a neonatal intensive care unit: predicting effectiveness with an artificial neural network model. Pharmacotherapy 2014;34:251-9.

24. Tulloh R, Medrano Lopez C, Checchia PA, Stapper C, Sumitomo $\mathrm{N}$, Gorenflo $\mathrm{M}$, et al. CHD and respiratory syncytial virus: global expert exchange recommendations. Cardiol Young 2017;27:1504-21.

25. Adult drug book-kidney disease program. Available from: https://kdpnet.kdp.louisville.edu/drugbook/adult/ [Last accessed on 13 Jun 2018]

26. European Medicines Agency. Available from: https://www.ema.europa.eu/en/search/search/field_ema_we b_categories\%253Aname_field/Human?search_api_views_fullte $\mathrm{xt}=$ drugs [Last accessed on 24 Mar 2019.

27. HAAD list of approved medications. Available from: https://www.haad.ae/haad/tabid/1328/Default.aspx [Last accessed on 08 Jun 2018].

28. Leibovici L, Yahav D, Paul M. Excess mortality related to cefepime. Lancet Infect Dis 2010;10:293-4.

29. Desrochers JF, Lemieux JP, Morin Belanger C, Paradis FS, Lord $\mathrm{A}$, Bell $\mathrm{R}$, et al. Development and validation of the PAIR (Pharmacotherapy Assessment in Chronic Renal Disease) criteria to assess medication safety and use issues in patients with CKD. Am J Kidney Dis 2011;58:527-35.

30. Pourrat X, Sipert AS, Gatault P, Sautenet B, Hay N, Guinard F, et al. Community pharmacist intervention in patients with renal impairment. Int J Clin Pharm 2015;37:1172-9.

31. Glatard A, Bourguignon L, Jelliffe RW, Maire P, Neely MN, Goutelle $\mathrm{S}$. Influence of renal function estimation on pharmacokinetic modeling of vancomycin in elderly patients. Antimicrob Agents Chemother 2015;59:2986-94.

32. Dooley MJ, Poole SG, Rischin D. Dosing of cytotoxic chemotherapy: impact of renal function estimates on the dose Ann Oncol 2013;24:2746-52.

33. Diego del Río E, Soy Muner D, Gratacos Santanach L, Ribas Sala J. The impact of different renal function measuring methods on the dosages of meropenem, piperacillin/tazobactam and cefepime in critically ill patients. Farm Hosp 2008;32:199-207.

34. Breton G, Froissart M, Janus N, Launay Vacher V, Berr $\mathrm{C}$, Tzourio $\mathrm{C}$, et al. Inappropriate drug use and mortality in community-dwelling elderly with impaired kidney function-the three-city population-based study. Nephrol Dial Transplant 2011;26:2852-9.

35. Lascano ME, Poggio ED. Kidney function assessment by creatinine-based estimation equations. Available from: www.clevelandclinicmeded.com/medicalpubs/diseasemanage ment/nephrology/kidney-function/. [Last accessed 11 Jan 2018]

36. Cockcroft DW, Gault MH. Prediction of creatinine clearance from serum creatinine. Nephron 1976;16:31-41.

37. Jones GRD. Estimating renal function for drug dosing decisions. Clin Biochem Rev 2011;32:81-8.

38. Karsch Volk M, Schmid E, Wagenpfeil S, Linde K, Heemann U, Schneider A. Kidney function and clinical recommendations of drug dose adjustment in geriatric patients. BMC Geriatr 2013;13:92-9.

39. Dersch D, McCormack J. Estimating renal function for drug dosing: rewriting the gospel? Can J Hosp Pharm 2008; 61:138-43. 